\title{
The Mechanism of Single Progesterone as Add-Back Therapy to GnRH-a Administration
}

\author{
Jiming Chen, Junling Liu, Weiwei Wei, Ying Cao, Yilin Sun, Huichao Xiao, Yafeng Zheng, Yunfen Jiang and \\ Ruxia Shi*
}

Department of Obstetrics and Gynecology, The Affiliated Changzhou NO. 2 People's Hospital of Nanjing Medical University, Changzhou, 213000, China

*Corresponding author: Ruxia Shi, Department of Obstetrics and Gynecology, The Affiliated Changzhou NO. 2 People's Hospital of Nanjing Medical University, No.68 Gehu Road, Wujin District, Changzhou, 213000, China.
Received Date: January 02, 2019

Published Date: January 04, 2019

\section{Opinion}

Endometriosis (EMS) is a common estrogen-dependent gynecological disorder, which affects quality of life and fertility of productive-aged women [1]. Current treatment of EMS is mainly based on surgery and post-operative maintenance treatment of ovarian suppressive agents. A major challenge for women with EMS is the post-operative recurrence [2-3]. Some medical treatments have been suggested for EMS, such as oral contraceptive pills, and gonadotropin-releasing hormone agonists (GnRH-a). GnRH-a is an important treatment modality for EMS, significantly reducing EMSrelated symptoms [4-6].

However, the employment of GnRH-a could reduce the estrogen level, leading to severe peri-menopausal symptoms such as hot flash, colpoxerosis, sexual hypoactivity, and bone loss, which hinders its long-term and extensive application [7]. The perimenopausal symptoms could be solved by the hormone based "addback therapy" [8-10]. As for add-back therapy, three regimens were recommended by Obstetrics and Gynecology Branch of Chinese Medical Association [11].

Estrogen and progesterone regimen: continuous combination of estrogen and progesterone. Estradiol valerate 0.5$1.5 \mathrm{mg} / \mathrm{d}$, or conjugated estrogen $0.3 \sim 0.45 \mathrm{mg} / \mathrm{d}$ or estradiol patch releasing $25 \sim 50 \mu \mathrm{g}$ daily, or estradiol gel $1.25 \mathrm{~g} / \mathrm{d}$ via percutaneous daub; for progesterone, $5 \mathrm{mg} / \mathrm{d}$ dydrogesterone or $2 \sim 4 \mathrm{mg} / \mathrm{d}$ medroxyprogesterone is often used. Compound preparation estradiol spironolone tablets can also be used, 1 tablet per day.

Single progesterone: $1.25 \sim 2.5 \mathrm{mg}$ norethindrone acetate daily.

Continuous use of tibolone, $1.25 \sim 2.5 \mathrm{mg} / \mathrm{d}$ is recommended.

According to the conventional understanding, the purpose of add-back to GnRH-a is to supplement estrogen to alleviate the series of problems caused by low estrogen. So why should we discuss this single progesterone regimen (In fact, this regimen has not been used too much clinically)? Many clinical teachers have asked this question in the gynecologic endocrine conference, this was a brief state of my personal understanding.

Before answering this question, let me briefly review the mechanism of add-back therapy to GnRH-a administration. The use of GnRH-a, which will inhibit the gonadal axis, is bound to result in patients' low estrogen status. In order to alleviate the series of problems caused by this low estrogen status, meanwhile to prolong patients' GnRH-a application time and compliance, based on the theory of "estrogen dosage window" (Different tissues have different sensitivity to estrogen), some scholars put forward a therapeutic regimen (Add-back) to keep estrogen at a normal level in the body so that it will not stimulate ectopic endometrial growth without causing perimenopausal symptoms and bone loss [E2:146 183 pmol/L (40 50 pg/ml)]. Such estrogen level does not affect curative effect and can reduce side effects [12].

Since it is to solve the problem of low estrogen, it is easy to understand the other two estrogen regimens: estrogen plus progesterone combination regimen (why to add progesterone dydrogesterone $5 \mathrm{mg}$ or MPA 2-4mg? EMS is an estrogen-dependent disease, the combination regimen is more conducive to endometrial atrophy, but there are also reports on single estrogen regimen); Use tibolone continuously with a recommended dose of 1.25 2.5 $\mathrm{mg} / \mathrm{d}$ (tibolone component: 7-methylenethinolone, its metabolites have three kinds of activities: estrogen, progesterone and androgen effects respectively, as a result, the compound effect is equivalent to the combination regimen.).

Some Gynecologists often feel confused regarding the single progesterone regimen (norethindrone acetate $1.25-2.5 \mathrm{mg}, \mathrm{qd}$ ) together with its mechanism. 
Before understanding this issue, we should first review the classification, function and characteristics of progesterone.

Progesterone is mainly divided into three categories:

1. Natural progesterone

2. Dydrogesterone: which is close to the natural progesterone

3. Synthetic progesterone: mostly progesterone derivatives or testosterone derivatives.

Synthetic progesterone is divided into four sub-categories:

1. $17 \alpha$ ' hydroxyprogesterone derivatives (representative drugs: medroxyprogesterone MPA, megestrol MA, cyproterone CPA)

2. 19-gestonorone derivatives (representative drug: nomegestrol)

3. spironolactone derivatives (drospirenone)

4. 19- nortestosterone derivatives (mainly including: norethindrone (NET) with estrane structure and norgestrel (LNG) with sterane structure).

As a derivative of 19-nortestosterone, norethindrone has an estrange structure. This derivative has high progesterone activity after metabolism, as well as androgen activity and partial estrogen activity. This characteristic determines that it has a good hemostatic effect on abnormal uterine bleeding (AUB) clinically. As we all know, synthetic progesterone has a very efficient and precise hemostatic effect, however, besides an effective progestogen effect, norethindrone has a synergistic effect to progestogen with its partial estrogen activity (via upregulation the activity of PR), moreover, the androgen activity of its metabolite at the same time, can reduce pelvic congestion effectively and the amount of uterine bleeding (Based on this principle, when progesterone injection is clinically used to treat AUB, 25-50mg testosterone propionate injection (androgen) via intramuscular injection qd is often given about 3-4 days before drug withdrawal to reduce withdrawal bleeding).

Based on the above statement and analysis, norethindrone selected in the single progesterone regimen of the add-back therapy should be obtained by taking the estrogen of its metabolites.

\section{Appendix}

Digression on synthetic progesterone from 19- nortestosterone derivatives.

Synthetic progesterone from 19- nortestosterone derivatives include: norethindrone (NET) which has an estrane structure and norgestrel (LNG) which has a sterane structure.

Norethindrone has been described previously and were briefly introduced here. (Note: As a synthetic progesterone preparation, norethindrone has an estrane structure, and it is a derivative of the synthetic 19-nortestosterone. It has weak estrogen activity, antiestrogen activity, together with mild androgen activity and protein assimilation effect, and its androgen activity is about $1 / 6$ of that of testosterone).
Levonorgestrel (LNG), which has a sterane structure, is the main component of the familiar Mirena intrauterine device and progesterone emergency contraception pills and is currently the most efficient progesterone. What is levonorgestrel (LNG)? LNG is a fully synthetic and highly effective progesterone (synthetic progesterone preparation which has a sterane structure). AS the optical active agent of racemic norgestrel, the progesterone activity of levonorgestrel is 1 time stronger than that of norgestrel and about 100 times that of norethindrone. Therefore, the dose can be halved compared with norgestrel, and the adverse reactions can be reduced correspondingly. Levonorgestrel mainly acts on hypothalamus and pituitary gland, thereby the peak levels of FSH and LH in the middle menstrual period will be significantly reduced or just disappear, and the ovulation ceases. Levonorgestrel has obvious anti-estrogen activity, which is about 10 times stronger than norethindrone. However, LNG has almost no estrogen activity, which can thicken cervical mucus and prevent sperm penetration. It shows strong progesterone activity on endometrial transformation, which can thin endometrium. Endometrium epithelial cells present low columnar shape and have poor secretion, which is not conducive to implantation of pregnant eggs (The contraceptive effect is accurate!). LNG also has certain androgen activity and protein assimilation effect, and both oral and subcutaneous injection can inhibit ovulation.

\section{Acknowledgement}

This work was supported by a grant from Major Science and Technology Project of Changzhou Health and Family Planning Commission(ZD201812).

\section{Conflict of Interest}

No conflict of interest.

\section{References}

1. Nirgianakis K, Bersinger NA, McKinnon B, Kostov P, Imboden S, et al. (2013) Regression of the inflammatory microenvironment of the peritoneal cavity in women with endometriosis by GnRHa treatment. Eur J Obstet Gynecol Reprod Biol 170(2): 550-554.

2. Almassinokiani F, Mehdizadeh A, Sariri E, Rezaei M, Almasi A, et al (2013) Effects of simvastatin in prevention of pain recurrences after surgery for endometriosis. Med Sci Monit 19: 534-539.

3. The Chinese medical association branch of obstetrics and gynecology endometriosis group (2007) The diagnosis and treatment of endometriosis. Chin J Obstet Gynecol 42 (9): 645-647.

4. Leng JH, Lang JH, Yang JX (2000) Progress of diagnosis and treatment of endometriosis. Chin J Obstet Gynecol 35 (1): 53-55.

5. Liu DY, Gu MJ, Shu JZ, Shi YX, Wang CY, et al. (2006) Effect of triptorelin and an extended-interval dosing regimen in the treatment of patients with endometriosis and adenomyoma. Chin J Obstet Gynecol 41 (10): 657-659.

6. Yang DZ and Wang MY (2010) Proceedings of the third national meeting on endometriosis and chronic pelvic pain. Chin J Obstet Gynecol 45 (4): 243-245.

7. Khan KN, Kitajima M, Hiraki K, Fujishita A, Nakashima M, et al. (2010) Cell proliferation effect of GnRH agonist on pathological lesions of women with endometriosis, adenomyosis and uterine myoma. Hum Reprod 25 (11): 2878-2890.

8. Wang YQ Zhang SF, Chen X, Zhu J, Hua KQ et al. (2009) Effects and safety of gonadotrophin-releasing hormone agonist combined with estradiol 
patch and oral medroxyprogesterone acetate on endometriosis. Chinese J Obstet Gynecol 44 (7): 7-9.

9. Liu PS, Li X, Liu Y, Hong-luan M, Yu-lan S (2008) Ximingting tablets in the treatment of women with climacteric syndrome. J Shandong Univ 46(8): 791-794.

10. Zhou L and Liu YJ (2011) Progress of black cohosh treatment for perimenopausal syndrome. Res Integ Tradit Chin West Med 3(3): 150-152.
11. Endometriosis Collaboration Group, Obstetrics and Gynecology Branch, Chinese Medical Association (2015). Chin J Obstet Gynecol 50(3): 161169.

12. Barbieri RL (1992) Hormone treatment of endometriosis: the estrogen threshold hypothesis. Am J Obster Gynecol 166(2): 740-745. 